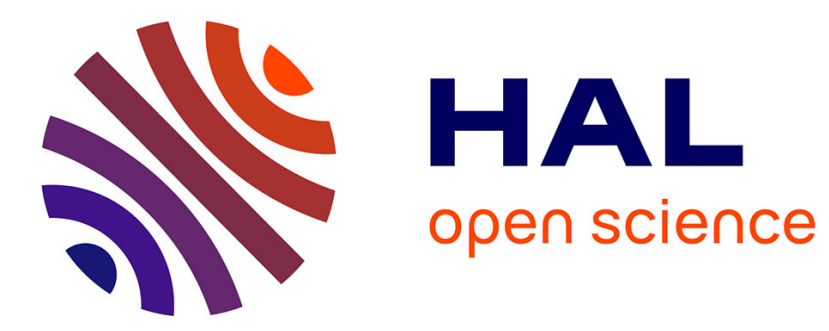

\title{
Un pyromètre rapide à plusieurs couleurs
}

R. Bouriannes, M. Moreau, J. Martinet

\section{To cite this version:}

R. Bouriannes, M. Moreau, J. Martinet. Un pyromètre rapide à plusieurs couleurs. Revue de Physique Appliquée, 1977, 12 (5), pp.893-899. 10.1051/rphysap:01977001205089300 jpa-00244256

\section{HAL Id: jpa-00244256 https://hal.science/jpa-00244256}

Submitted on 1 Jan 1977

HAL is a multi-disciplinary open access archive for the deposit and dissemination of scientific research documents, whether they are published or not. The documents may come from teaching and research institutions in France or abroad, or from public or private research centers.
L'archive ouverte pluridisciplinaire HAL, est destinée au dépôt et à la diffusion de documents scientifiques de niveau recherche, publiés ou non, émanant des établissements d'enseignement et de recherche français ou étrangers, des laboratoires publics ou privés. 
Classification

Physics Abstracts

0.620

\title{
UN PYROMÈTRE RAPIDE A PLUSIEURS COULEURS
}

\author{
R. BOURIANNES et M. MOREAU
}

Laboratoire d'Energétique et de Détonique $\left({ }^{*}\right)$

et J. MARTINET

Laboratoire de Thermique (**), Ecole Nationale Supérieure de Mécanique et d'Aérotechnique, rue Guillaume VII, 86034 Poitiers Cedex, France

(Reçu le 20 décembre 1976, accepté le 18 janvier 1977)

\begin{abstract}
Résumé. - Description d'un pyromètre optique au temps de montée de l'ordre de 50 nanosecondes et permettant la mesure de 4 températures de luminance, dans le visible et le proche infrarouge, d'une surface de $4 \mathrm{~mm}^{2}$ et de température supérieure à $1700 \mathrm{~K}$.

Le pyromètre est utilisé, à titre d'exemple, pour mesurer la température d'un élément de graphite puis celle des produits de détonation d'un explosif liquide.
\end{abstract}

Abstract. - A fast optical pyrometer is described; its rise time is about $50 \mathrm{~ns}$; it gives for a $4 \mathrm{~mm}^{2}$ surface at a temperature higher than $1700 \mathrm{~K}$, four brightness temperature in the visible and in the near infrared.

This pyrometer has been used to measure the temperature of a graphite sample and of the detonation products of a liquid explosive.

1. Introduction. - Un matériau, siège d'un phénomène physique mettant en jeu durant des temps très brefs (de l'ordre de quelques nanosecondes) de grandes quantités d'énergie, peut, durant ce laps de temps, se voir porté à une température très élevée pouvant atteindre plusieurs milliers de degrés. C'est le cas lors de la détonation d'explosifs, les produits résultants étant alors portés à $3000 \mathrm{~K}$ environ en moins de $50 \mathrm{~ns}$. Il en est de même lors de l'impact d'un faisceau d'électrons sur une cible ou encore lors du passage d'une décharge électrique intense dans un élément résistant.

C'est plus particulièrement en vue d'une application à ce dernier exemple que nous avons réalisé le pyromètre optique décrit ci-après, lequel, pour pallier à une connaissance insuffisante du facteur d'émission du matériau chauffé, a été conçu pour travailler dans quatre longueurs d'onde différentes (ce qui permet éventuellement la détermination des températures à deux et trois couleurs) et ce, avec des temps de réponse de l'ordre de quelques dizaines de nanosecondes, ce laps de temps étant néanmoins supposé suffisant pour que soient satisfaites les conditions classiques d'équilibre thermodynamique en dehors desquelles une température ou un facteur d'émission ne peuvent être définis.

(*) L. A. 193 .

(**) L. A. 191.
2. Les températures de couleur. Définitions. Mesures. - 2.1 LA TEMPÉRATURE A DEUX COUleURS. - On appelle [1 et 2] température à deux couleurs $T_{2 \mathrm{c}}$ relativement aux longueurs d'onde $\lambda_{1}$ et $\lambda_{2}$ d'une surface émettrice de température absolue vraie $T$ et de facteurs monochromatiques normaux d'émission $\varepsilon\left(\lambda_{1}, T\right)$ et $\varepsilon\left(\lambda_{2}, T\right)$, la température $T_{2 \mathrm{c}}$ du corps noir pour laquelle le quotient :

$$
\frac{L^{0}\left(\lambda_{1}, T_{2 c}\right)}{L^{0}\left(\lambda_{2}, T_{2 c}\right)}
$$

de ses luminances monochromatiques $L^{0}$ relativement aux longueurs d'onde $\lambda_{1}$ et $\lambda_{2}$ est égal au quotient :

$$
\frac{L\left(\lambda_{1}, T\right)}{L\left(\lambda_{2}, T\right)}
$$

des luminances monochromatiques normales $L$ de la surface émettrice.

L'application, supposée possible, de la loi de Wien, conduit à la relation suivante reliant $T$ et $T_{2 \mathrm{c}}$ :

$$
\frac{1}{T}-\frac{1}{T_{2 \mathrm{c}}}=\frac{L_{\mathrm{n}}\left[\frac{\varepsilon\left(\lambda_{1}, T\right)}{\varepsilon\left(\lambda_{2}, T\right)}\right]}{C_{2}\left(\frac{1}{\lambda_{1}}-\frac{1}{\lambda_{2}}\right)}
$$

où $C_{2}$ désigne la constante classique $(14,388 \mu \mathrm{mK})$; le cas particulier $\varepsilon\left(\lambda_{1}, T\right) \simeq \varepsilon\left(\lambda_{2}, T\right)$ conduit naturellement à l'approximation $T \simeq T_{2 \mathrm{C}}$. 
2. 2 LE PYROMÈTRE A DEUX COULEURS. - Dans son aspect de principe, le pyromètre à deux couleurs et à comparaison des luminances - le seul envisagé ici - a tout d'abord pour rôle de concentrer les énergies correspondant aux luminances monochromatiques normales $L\left(\lambda_{1}, T\right)$ et $L\left(\lambda_{2}, T\right)$ sur deux détecteurs identiques, en l'occurrence des photodétecteurs dont la fonction consiste à délivrer des tensions électriques $V_{1}$ et $V_{2}$ reliées aux énergies $E_{1}$ et $E_{2}$ reçues par une relation linéaire du type :

$$
E=a V+v
$$

où $a$ et $v$ sont des constantes caractéristiques de chaque voie de mesure. En général, la tension d'obscurité $v$ peut être rendue suffisamment petite pour pouvoir être considérée comme négligeable devant $a V$. Dans ces conditions, les tensions $V_{1}$ et $V_{2}$ délivrées sont respectivement proportionnelles aux énergies $E_{1}$ et $E_{2}$ reçues :

$$
E_{1}=a_{1} V_{1}, \quad E_{2}=a_{2} V_{2},
$$

la mesure des tensions $V_{1}^{0}$ et $V_{2}^{0}$ délivrées lors de la visée sur un corps noir à la température $T_{0}$, et qui vérifient donc la relation :

$$
E_{1}^{0}=a_{1} V_{1}^{0}, \quad E_{2}^{0}=a_{2} V_{2}^{0}
$$

permettant le calcul des constantes $a_{1}$ et $a_{2}$.

L'application de la loi de Wien conduit finalement à la relation :

$$
\frac{1}{T}-\frac{1}{T_{0}}=\frac{L_{\mathrm{n}}\left[\frac{V_{1}}{V_{1}^{0}} / \frac{V_{2}}{V_{2}^{0}}\right]}{C_{2}\left(\frac{1}{\lambda_{1}}-\frac{1}{\lambda_{2}}\right)}
$$

L'étalonnage du pyromètre à deux couleurs ne nécessite donc qu'un seul point de mesure (visée sur un corps noir de température $T^{0}$, entraînant la connaissance de $V_{1}^{0}$ et $\left.V_{2}^{0}\right)$.

2.3 LA TEMPÉRATURE A TROIS COULEURS. - Elle se définit de la même manière que la température de deux couleurs, mais cette fois à partir de trois luminances monochromatiques normales, des facteurs monochromatiques normaux d'émission et du rapport :

$$
R=\frac{L\left(\lambda_{1}, T\right) \cdot L\left(\lambda_{3}, T\right)}{\left(L\left(\lambda_{2}, T\right)\right)^{2}}
$$

que l'on pose égal au rapport $R^{0}$ correspondant et relatif au corps noir à la température $T_{3 \mathbf{c}}^{1,2,3}$, laquelle est, par définition, la température à trois couleurs $\left(\lambda_{1}, \lambda_{2}, \lambda_{3}\right)$; cette dernière peut donc être rattachée à la température vraie $T$ par la relation :

$$
\frac{1}{T}-\frac{1}{T_{3 \mathrm{c}}}=\frac{L_{\mathrm{n}}\left[\frac{\varepsilon\left(\lambda_{1}, T\right) . \varepsilon\left(\lambda_{3}, T\right)}{\left(\varepsilon\left(\lambda_{2}, T\right)\right)^{2}}\right]}{C_{2}\left(\frac{1}{\lambda_{1}}+\frac{1}{\lambda_{3}}-\frac{2}{\lambda_{2}}\right)},
$$

le cas particulier $\varepsilon\left(\lambda_{1}, T\right) \cdot \varepsilon\left(\lambda_{3}, T\right) \simeq\left[\varepsilon\left(\lambda_{2}, T\right)\right]^{2}$ conduit à l'approximation $T \simeq T_{3 \mathrm{c}}^{1,2,3}$ qui se rencontre notamment $[3,4]$ lorsque :

- d'une part les trois longueurs d'onde $\lambda_{1}, \lambda_{2}$ et $\lambda_{3}$ sont choisies telles que $2 \lambda_{2}=\lambda_{1}+\lambda_{3}$,

- d'autre part le facteur monochromatique normal d'émission peut, au voisinage de sa valeur intermédiaire $\varepsilon\left(\lambda_{2}, T\right)$, être assimilé à une fonction linéaire de $\lambda$, de faible variation relative dans les intervalles $\lambda_{2}-\lambda_{1}$, $\lambda_{3}-\lambda_{2}$.

2.4 LE PYROMÈTRE A TROIS COULEURS. - Basé sur le même principe que le pyromètre à deux couleurs, il comporte trois voies opto-électriques de mesure aussi semblables que possible. Son étalonnage s'effectue comme précédemment et ne nécessite ainsi qu'un seul point de mesure (visée sur un corps noir de température $T^{0}$, entraînant la connaissance de $V_{1}^{0}, V_{2}^{0}$ et $V_{3}^{0}$ ), la température $T$ étant alors donnée par la relation :

$$
\frac{1}{T}-\frac{1}{T^{0}}=\frac{L_{\mathrm{n}}\left[\frac{V_{1}}{V_{1}^{0}} \cdot \frac{V_{3}}{V_{3}^{0}} /\left(\frac{V_{2}}{V_{2}^{0}}\right)^{2}\right]}{C_{2}\left(\frac{1}{\lambda_{1}}+\frac{1}{\lambda_{3}}-\frac{2}{\lambda_{2}}\right)} .
$$

3. Aspect pratique. - 3.1 GÉNÉRALITÉS. - Différents types de pyromètres à deux et trois couleurs ont déjà été réalisés précédemment dans le cadre des principes qui viennent d'être exposés. Certains utilisent un dispositif mécanique de commutation entre les voies ; le temps de réponse est alors assez élevé (de l'ordre de $10^{-2} \mathrm{~s}$ ). Dans d'autres types d'appareils, la séparation du faisceau en plusieurs voies est réalisée au moyen d'un spectrographe $[5,7]$ ou de fibres optiques $[6,8]$, un système optique faisant ensuite converger chacun des faisceaux lumineux résultant sur un photomultiplicateur. Le temps de réponse peut alors être plus faible (de l'ordre de $10^{-8} \mathrm{~s}$, pour certaines réalisations récentes).

3.2 LE PYROMÈTRE ET SA CHAINE DE MESURE (Figs. 1 et 2). - L'appareil dont nous nous sommes proposés la réalisation devait, avant tout, répondre à des impératifs de simplicité de réalisation et de mise en œuvre,

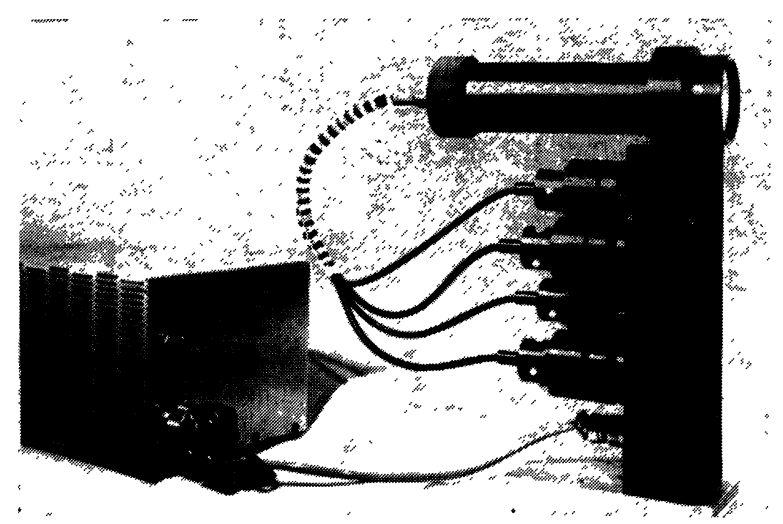

FIG. 1. - Le pyromètre optique à 4 couleurs. [The four colour optical pyrometer.] 


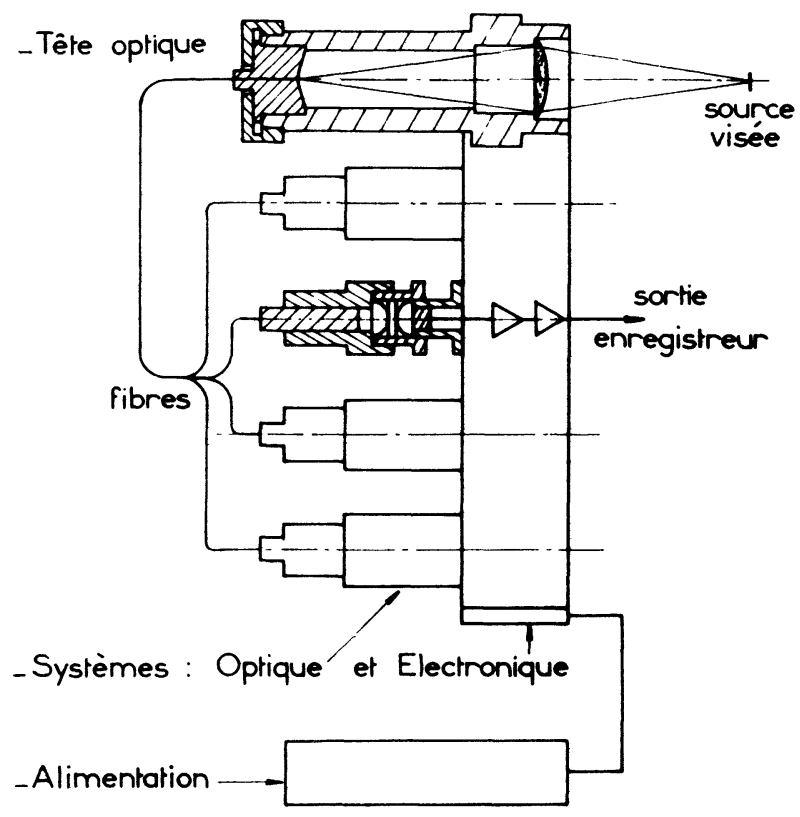

Fig. 2. - Schéma de principe du pyromètre.

[Schematic design of the pyrometer.]

ce qui nous a conduit à écarter d'une part les spectrographes pour des raisons d'encombrement et d'autre part les photomultiplicateurs, fragiles et nécessitant une installation importante pour leur alimentation, au profit de chaînes opto-électroniques constituées de fibres optiques, de filtres interférentiels et de photodiodes (ces dernières présentant de plus une bonne sensibilité dans le proche infra-rouge).

Outre une lentille objectif commune, laquelle forme sur les extrémités jointives de quatre fibres optiques de diamètre individuel $1 \mathrm{~mm}$ l'image de la surface visée, de diamètre total $4 \mathrm{~mm}$, située à $285 \mathrm{~mm}$ de la lentille, chaque voie comporte :

- un système optique constitué d'une des quatre fibres laquelle, par l'intermédiaire d'un dispositif comportant deux lentilles convergentes placées de part et d'autre d'un filtre interférentiel, transmet le signal lumineux au photodétecteur, les longueurs d'onde des filtres interférentiels $\left(\lambda_{1}=0,650 ; \lambda_{2}=0,783\right.$; $\lambda_{3}=0,915 ; \lambda_{4}=1,008 \mu \mathrm{m}$ ) ont été choisies de manière à satisfaire au mieux les conditions :

$$
2 \lambda_{2} \simeq \lambda_{1}+\lambda_{3} \text { et } 2 \lambda_{3} \simeq \lambda_{2}+\lambda_{4}
$$

ce qui, en supposant vérifiée la condition relative à la variation du facteur d'émission (cf. $\S 2.3$ ), permet le calcul des températures à 3 couleurs $T_{3 \mathbf{c}}^{1,2,3}$ et $T_{3 \mathbf{c}}^{2,3,4}\left({ }^{1}\right)$.

Un système électronique comportant :

- un photodétecteur au silicium type RCA C 30815 dont le maximum de sensibilité se situe à $0,9 \mu$ et dont

(1) Température notée plus simplement $T_{3 \mathrm{c}}$ et non $T_{3 \mathrm{c}}^{i, j, k}$ lorsqu'il n'y a pas d'ambiguité sur la numérotation des voies de mesure considérées. le temps de montée est de $35 \mathrm{~ns}$. Sa faible surface $\left(1 \mathrm{~mm}^{2}\right)$ permet la visée de sources de petites dimensions ;

- un amplificateur de tension adjoint au précédent ;

- un amplificateur de puissance qui permet d'une part une utilisation de l'ensemble en continu, tout en conservant le même temps de montée et d'autre part de ramener à zéro le courant d'obscurité du photodétecteur.

\section{Deux pyromètres ont été réalisés :}

- le premier (Fig. 1) dans lequel les ensembles optique et électronique sont solidaires et les fibres optiques courtes $(350 \mathrm{~mm})$,

- le second dans lequel ces ensembles sont disjoints et les fibres optiques longues ce qui (dans le cas où apparaissent des phénomènes électriques parasites fréquemment provoqués par le dispositif de chauffage du matériau étudié) permet d'éloigner et par là même de protéger plus facilement le système électronique.

3. 3 L'ÉTALONNAGE EN TEMPÉRATURE. - Un premier étalonnage destiné à évaluer les limites de fonctionnement a été effectué en visant une lampe à ruban de tungstène dont la température dans le rouge a été mesurée à l'aide d'un pyromètre monochromatique classique, la visée s'effectuant à travers un hublot de silice. La température de luminance de cette lampe a ensuite été déterminée pour les quatre longueurs d'onde retenues précédemment, à l'aide des valeurs du facteur d'émission relevées dans la bibliographie [9 et 10].

Entre les températures vraies de $1700 \mathrm{~K}$ (limite inférieure d'utilisation du pyromètre) et $2500 \mathrm{~K}$ (limite supérieure de fonctionnement de la lampe), nous avons, pour chacune des quatre longueurs d'onde de travail, vérifié la linéarité de la relation liant les énergies aux tensions électriques délivrées (la figure 3

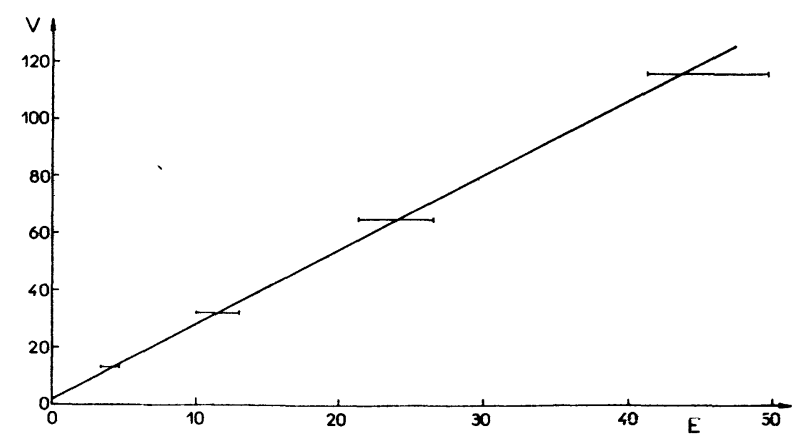

Fig. 3. - Tension électrique délivrée $V$ (en $\mathrm{mV}$ ), fonction de l'énergie reçue $E$ (unités arbitraires), sur la voie $2\left(\lambda_{2}=0,783 \mu \mathrm{m}\right)$.

[Output voltage $V(\mathrm{mV})$ versus incident energy $E$ (arbitrary units) for path $2\left(\lambda_{2}=0,783 \mu \mathrm{m}\right)$.]

traduit en particulier les résultats obtenus avec la seconde voie correspondant à la longueur d'onde, $\left.\lambda_{2}=0,783 \mu \mathrm{m}\right)$. 
Ceci étant, pour la température vraie $T^{0}=2459 \mathrm{~K}$, nous avons obtenu :

- sur la voie $1: \lambda_{1}=0,650 \mu \mathrm{m}, \quad V_{1}^{0}=140 \mathrm{mV}$

- sur la voie $2: \lambda_{2}=0,783 \mu \mathrm{m}, \quad V_{2}^{0}=143 \mathrm{mV}$

- sur la voie $3: \lambda_{3}=0,915 \mu \mathrm{m}, \quad V_{3}^{0}=172 \mathrm{mV}$

- sur la voie $4: \lambda_{4}=1,008 \mu \mathrm{m}, \quad V_{4}^{0}=127 \mathrm{mV}$

d'où les relations suivantes, lesquelles permettent de relier les différentes températures de couleur aux tensions délivrées (exprimées respectivement en Kelvin et en millivolts) :

- Température de luminance (sur la voie 1 par exemple) :

$$
\frac{10^{4}}{T_{1}^{1}}=6,31-0,45 L_{\mathrm{n}}(V) .
$$

- Température de deux couleurs (sur les voies 2 et 3 par exemple)

$$
\frac{10^{4}}{T_{2 \mathrm{c}}^{2,3}}=3,38-3,77 L_{\mathrm{n}}\left(V_{2} / V_{3}\right) .
$$

- Température de trois couleurs (sur les voies 1,2 et 3 par exemple)

$$
\frac{10^{4}}{T_{3 \mathrm{c}}^{1,2,3}}=5,60-9,02 L_{\mathrm{n}}\left(\frac{V_{1} \cdot V_{3}}{V_{2}^{2}}\right) \text {. }
$$

3.4. LeS ERREURS DE MESURE. - Lorsque, ne connaissant pas les valeurs du facteur monochromatique normal d'émission dans la bande spectrale retenue pour la mesure, on utilise les résultats fournis par les quatre voies, on peut, lors de la détermination de la température à deux ou trois couleurs, introduire des erreurs de deux sortes dues essentiellement d'une part à la variation anormale du facteur d'émission avec la longueur d'onde, cette variation ne permettant pas le maintien des hypothèses rappelées ( $§ 2.1$ et 2.3 ) lors des définitions des températures et d'autre part à la non-uniformité de la température de la surface visée. Sans revenir sur le calcul de ces erreurs dans le cas général $[4,11,12]$, nous allons envisager succinctement les erreurs qui s'introduisent dans l'un et l'autre cas.

Supposons la température vraie égale à $2800 \mathrm{~K}$; avec la méthode à deux couleurs $\left(\lambda_{1}=0,650 \mu \mathrm{m}\right.$, $\left.\lambda_{2}=0,783 \mu \mathrm{m}\right) \mathrm{si}$

$\frac{\varepsilon\left(\lambda_{1}, T\right)}{\varepsilon\left(\lambda_{2}, T\right)}=1,96($ au lieu de 1$)$, alors : $T_{2 \mathrm{c}}^{1,2}=5600 \mathrm{~K} ;$

avec la méthode à trois couleurs $\left(\lambda_{1}=0,650 \mu \mathrm{m}\right.$, $\left.\lambda_{2}=0,783 \mu \mathrm{m}, \lambda_{3}=0,915 \mu \mathrm{m}\right)$

$$
\left.\frac{\varepsilon\left(\lambda_{1}, T\right) \cdot \varepsilon\left(\lambda_{3}, T\right)}{\left(\varepsilon\left(\lambda_{2}, T\right)\right)^{2}}=1,22 \text { (au lieu de } 1\right)
$$

conduirait à la même valeur de la température de trois couleurs $T_{3 \mathbf{c}}^{1,2,3}(5600 \mathrm{~K})$, ce qui est inacceptable.
Encore que ces cas extrêmes soient peu probables (l'étroitesse de la bande spectrale de mesure impliquant pour les facteurs monochromatiques des valeurs très voisines), ils ne sont pas à exclure totalement du fait d'une modification de l'état de surface durant le temps nécessaire à la mesure ;

- Supposons maintenant que les quatre voies de mesure ne visent pas la même zone de la surface du matériau étudié; on peut se demander quelle est l'erreur introduite dans le cas où ces quatre surfaces n'ont pas rigoureusement la même température, celle-ci variant localement d'une petite valeur $\mathrm{d} T$ autour d'une valeur moyenne $T$, tout en supposant maintenues les hypothèses relatives aux différents facteurs d'émission dont la variation n'est pas ici à l'origine d'erreurs supplémentaires.

Avec la méthode à deux couleurs, si $T_{1}$ et $T_{2}$ sont les températures des surfaces visées, la température à 2 couleurs $T_{2 \mathrm{c}}^{1,2}$ se définit pratiquement au moyen de l'égalité (cf. § 2.1) :

$$
\frac{L\left(\lambda_{1}, T_{1}\right)}{L\left(\lambda_{2}, T_{2}\right)}=\frac{L^{0}\left(\lambda_{1}, T_{1,2}\right)}{L^{0}\left(\lambda_{2}, T_{1,2}\right)} .
$$

Si $\quad T_{1}=T-\mathrm{d} T, T_{2}=T+\mathrm{d} T$, la relation (11) conduit à l'erreur $\Delta T=T-T_{2 \mathrm{c}}^{1,2}$ affectant la mesure de la température moyenne $T$, laquelle s'écrit :

$$
\frac{\Delta T}{T_{2 \mathrm{c}}^{1,2}}=\frac{\lambda_{2}+\lambda_{1}}{\lambda_{2}-\lambda_{1}} \frac{\mathrm{d} T}{T}=11 \frac{\mathrm{d} T}{T} \text { dans le cas présent } .
$$

Avec la méthode à trois couleurs, un raisonnement analogue conduit, dans le cas où $T_{1}=T_{2}=T+\mathrm{d} T$, $T_{3}=T-\mathrm{d} T$ à l'erreur $\Delta T=T-T_{3 \mathrm{c}}^{1,2,3}$, laquelle' s'écrit :

$$
\begin{aligned}
\frac{\Delta T}{T_{3 \mathrm{c}}^{1,2,3}} & =\frac{-\frac{1}{\lambda_{1}}+\frac{1}{\lambda_{3}}+\frac{2}{\lambda_{2}} \frac{\mathrm{d} T}{T}}{\frac{1}{\lambda_{1}}+\frac{1}{\lambda_{3}}-\frac{2}{\lambda_{2}}} \\
& =28 \frac{\mathrm{d} T}{T} \text { dans le cas présent }
\end{aligned}
$$

Ces résultats donnent un aperçu des limites des techniques de mesure à deux et trois couleurs dès lors que l'on s'écarte du cas idéal ; d'où la nécessité impérieuse de bien préciser les conditions expérimentales.

3.5 L'ÉTAlonNage EN TEMPS DE RÉPONSE. - Par définition, le temps de réponse est celui qui s'écoule entre le moment où le pyromètre reçoit un signal lumineux et celui où il délivre un signal électrique d'amplitude correspondante. Ce temps se décompose en deux parties (Fig. 4a), à savoir :

- le temps dit de transit $\Delta t_{1}$ qui s'écoule entre le moment de l'impact du signal optique sur le détecteur et celui où débute le signal électrique correspondant,

- le temps dit de montée $\Delta t_{2}$ qui s'écoule entre le début et la fin de la montée de ce dernier signal (plus 


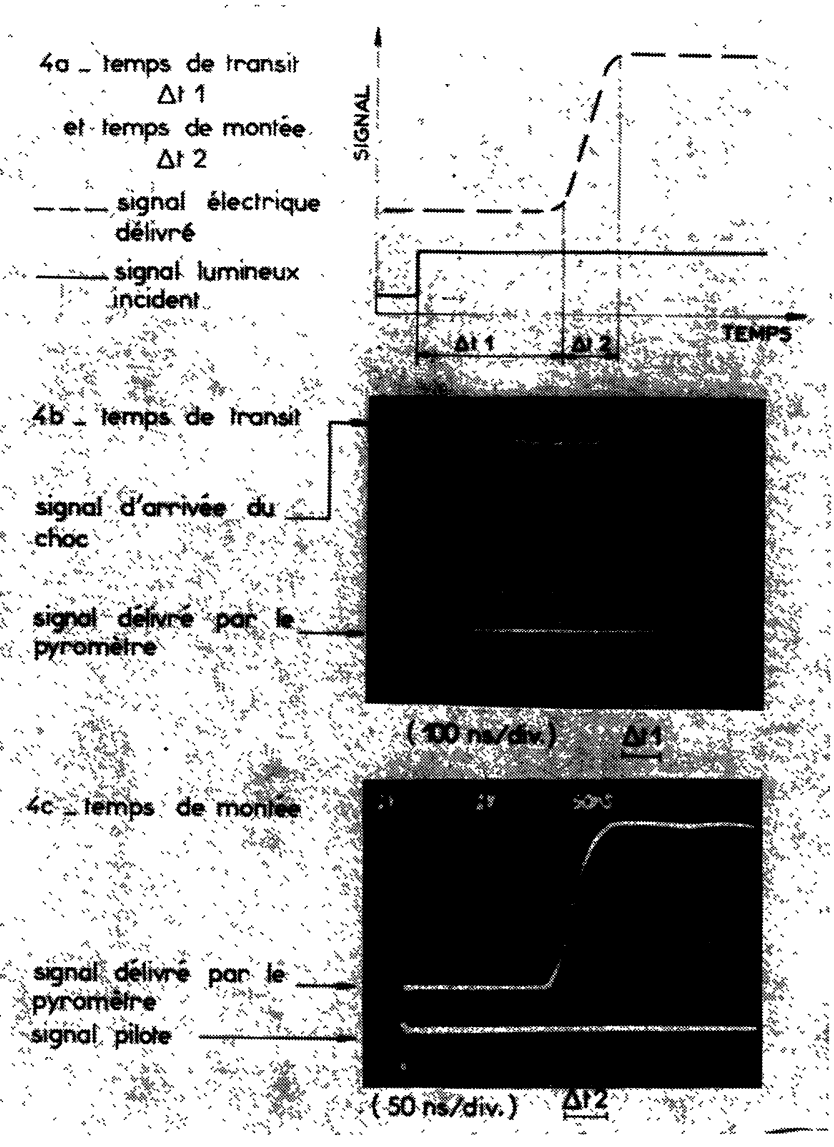

Fig. 4. - Détermination du temps de réponse : $4 a$ ) temps de transit $\Delta t_{1}$ et temps de montée $\Delta t_{2} ; 4 b$ ) temps de transit (échelle des temps $100 \mathrm{~ns} /$ div.) ; 4c) temps de montée (échelle des temps $50 \mathrm{~ns} /$ div.).

[Response time measurement : $4 a$ ) transit time $\Delta t_{1}$ and rise time $\Delta t_{2} ; 4 b$ ) transit time (time scale $100 \mathrm{~ns} / \mathrm{div}$. ) ; $4 c$ ) rise time (time scale $50 \mathrm{~ns} / \mathrm{div}$.$) .]$

précisément entre le moment où le signal atteint $10 \%$ de la valeur d'équilibre et celui où il atteint $90 \%$ de cette même valeur).

En général, le temps de transit d'un système électronique est inférieur à $100 \mathrm{ns,} \mathrm{donc} \mathrm{négligeable} \mathrm{devant} \mathrm{le}$ temps de montée supérieur à la milliseconde de la plupart des pyromètres. Or dans le cas présent ces deux temps sont du même ordre. Vu l'importance que présentait leur estimation précise, une détermination soignée sur chacune des quatre voies de mesure s'est avérée indispensable. Les résultats n'ont d'ailleurs pas sensiblement différé d'une voie à l'autre. Aussi ne présentons nous ci-après que ceux relatifs à une voie :

Le temps $\Delta t_{1}$ de transit a été mesuré de la manière suivante : une impulsion lumineuse, dont l'origine était repérée dans le temps, était obtenue en ionisant un petit volume d'argon au moyen d'une onde de choc intense (H. Presles, L. A. 193, communication privée, 1976). L'arrivée de l'onde était repérée par un signal électrique convenable et l'impulsion lumineuse délivrée quelques nanosecondes plus tard (cette durée, liée à celle d'ionisation du gaz étant par ailleurs bien infé- rieure à celle introduite par la lecture des enregistrements oscillographiques sur la détermination du temps $\left.\Delta t_{1}\right)$. Cette impulsion lumineuse était recueillie par la fibre optique puis transmise au photodétecteur, lequel la transformait en signal électrique ; ce dernier était ensuite observé conjointement avec le signal précédent sur le même oscillogramme (Fig. 4b). Leur décalage dans le temps représentant le temps de transit du pyromètre. L'examen de l'enregistrement obtenu montre que :

$$
\Delta t_{1}=95 \pm 5 \mathrm{~ns} .
$$

Le temps $\Delta t_{2}$ de montée n'a pu, quant à lui, être mis en évidence avec suffisamment de précision avec le dispositif ci-dessus car la luminosité de l'argon n'était pas constante, mais au contraire variait au fur et à mesure de la progression de l'onde de choc dans ce gaz. On a donc procédé à l'enregistrement, au moyen du pyromètre, du signal lumineux issu d'un laser à argon (type Spectra Physics 164) piloté par un signal carré. Le signal pilote et celui délivré par le pyromètre (ce dernier intégrant les temps de toute la chaîne de mesure) ont été enregistrés sur le même oscillogramme (Fig. 4c). L'examen de ce dernier montre que le temps de montée $\Delta t_{2}$ est bien inférieur à $50 \pm \mathrm{ns}$; quant au temps de transit (200 ns) visible sur cet oscillogramme, c'est la somme de celui relatif au système laser (lequel est inconnu) et de celui relatif au pyromètre.

En résumé, le temps de réponse du pyromètre peut donc être estimé à $\Delta t_{1}+\Delta t_{2}=145 \pm 10 \mathrm{~ns}$. Le temps de transit étant, par ailleurs connu et estimé à $95 \mathrm{ns,}$ le pyromètre peut, en pratique, être exploité avec un temps de montée de 50 ns.

4. Exemples d'application. - 4.1. GÉNÉRALITÉS. L'appareil décrit ci-dessus a été, dans un premier stade, utilisé pour mesurer la température d'une cible de graphite à structure fine (type Carbone Lorraine 730 comprimé) chauffée par effet Joule. Dans un second stade, nous avons mis à profit les possibilités de ce même appareil du point de vue temps de montée, en étudiant l'évolution de la température des produits de la détonation d'un explosif.

4.2 Mesure DE LA TEMPÉRATURe D'UNE CIBLE DE GRAPHITE. - L'élément étudié de dimensions $8 \times 5 \mathrm{~mm}$ et d'épaisseur $1 \mathrm{~mm}$ était placé dans une enceinte à vide, puis porté par effet Joule sous l'effet d'un courant de $90 \mathrm{~A}$, à une température de l'ordre de $3000 \mathrm{~K}$, le vide étant alors limité par la seule tension de vapeur du graphite.

L'enregistrement en fonction du temps des tensions de sortie des quatre voies de mesure du pyromètre a été réalisé sur un oscilloscope (Fig. 5). A partir des quatre tensions $V_{i}$ délivrées une fois le régime stationnaire atteint, soit $4 \mathrm{~s}$ environ après le début du chauffage (Fig. 6), on a procédé au calcul des quatre températures de luminance $T_{\mathrm{i}}$. En prenant pour le facteur monochromatique normal d'émission du graphite la 


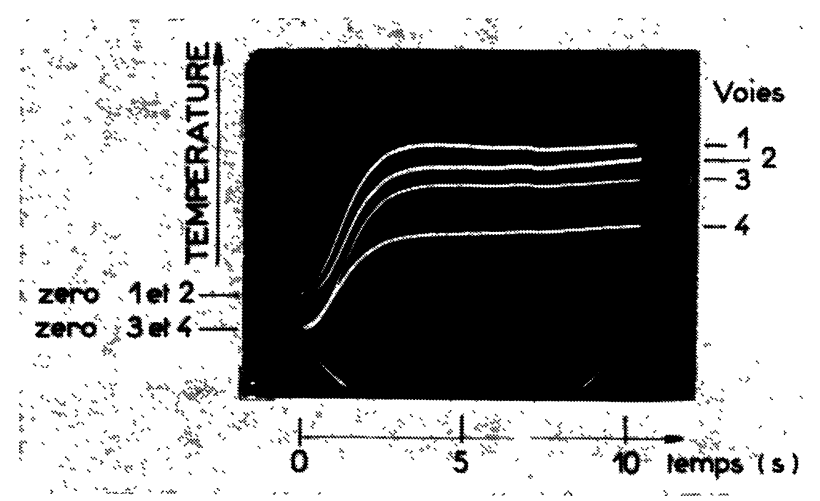

Fig. 5. - Evolution de la température d'une cible en graphite.

[Temperature of a graphite sample versus time.]

\begin{tabular}{|c|c|c|c|c|}
\hline Voie & 1 & 2 & 3 & 4 \\
\hline $\begin{array}{c}\text { Longueur } d^{\prime} \text { onde } \\
\lambda(\mu \mathrm{m})\end{array}$ & 0,650 & 0,783 & 0,915 & 1,008 \\
\hline Tensions $(\mathrm{mV})$ & 468 & 398 & 443 & 292 \\
\hline $\begin{array}{c}\text { Tempéralure de } \\
\text { luminance }(K)\end{array}$ & 2830 & 2840 & 2875 & 2860 \\
\hline $\begin{array}{c}\text { Tempéralure } \\
\text { vraie T }(K)\end{array}$ & 2850 & 2860 & 2900 & 2890 \\
\hline
\end{tabular}

$T=2875 \pm 25 \mathrm{~K}$

Fig. 6. - Températures de luminance d'une cible en graphite après $4 \mathrm{~s}$ de chauffage.

[Brightness temperature of a graphite sample heated for $4 \mathrm{~s}$.]

valeur supposée constante 0,95 [13], on en déduit les températures vraies des quatre surfaces visées.

On peut constater (Fig. 6) que $T=2875 \pm 25 \mathrm{~K}$. Le calcul des températures à 2 et 3 couleurs donne :

$$
\begin{aligned}
& T_{1,2}=2780 \mathrm{~K} \quad \text { (erreur relative } 3,4 \% \text { ) } \\
& T_{2,3}=2640 \mathrm{~K} \quad \text { (erreur relative } 9 \% \text { ) } \\
& T_{1,2,3}=3170 \mathrm{~K} \quad \text { (erreur relative } 10 \% \text { ) }
\end{aligned}
$$

Il est évident que dans cet exemple où le facteur d'émission est relativement bien connu et voisin de l'unité, les températures de luminance sont proches de la température vraie. Par contre, les erreurs relatives importantes sur les températures à deux couleurs, plus importantes encore sur celles à trois couleurs, peuvent s'expliquer par le fait que l'ensemble des zones visées (cercle de $4 \mathrm{~mm}$ de diamètre sur élément de graphite de $8 \times 5 \mathrm{~mm}$ ) n'est pas, du fait des effets de bord, à température uniforme.

4.3 MESURE DE LA TEMPÉRATURE DES PRODUITS DE DÉTONATION D'UN EXPLOSIF CONDENSÉ. - Le mélange explosif (Nitrométhane additionné de polymétacrylate de méthyle et d'aluminium) était contenu dans un tube horizontal, de $100 \mathrm{~mm}$ de long et $18 \mathrm{~mm}$ de diamètre, la détonation se propageant de l'arrière vers l'avant du tube, fermé par une paroi en verre.

Le pyromètre mesurait l'élévation de température, sous l'effet de la détonation, des produits situés immédiatement avant cette paroi. L'enregistrement a été effectué sur une seule voie, dans le rouge $(\lambda=0,65 \mu \mathrm{m})$. Bien que l'examen détaillé de l'oscillogramme obtenu (Fig. 7) sorte du cadre de cette étude (l'expérience

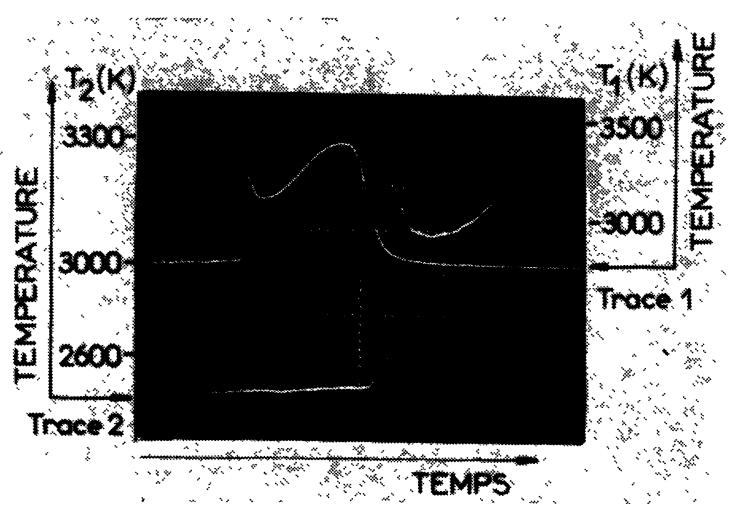

Fig. 7. - Evolution de la température de luminance des produits de la détonation d'un mélange de nitrométhane-altuglass et aluminium (trace 1:500 ns/div.-trace 2: $200 \mathrm{~ns} /$ div.).

[Brightness temperature of the detonation products of a nitromethane-plexiglass-aluminum mixture (trace : $1500 \mathrm{~ns} /$ div.trace $2: 200 \mathrm{~ns} / \mathrm{div}$.]

présentée ayant pour but principal de tester les possibilités du pyromètre) nous avons pu néanmoins faire les remarques suivantes :

- la montée du signal, fonction de l'augmentation de température des produits derrière le front de détonation s'effectue en 55 ns environ;

- à cet instant, la température de luminance des produits en combustion est de $3210 \mathrm{~K}$.

L'étude complète de l'enregistrement obtenu, notamment en ce qui concerne l'évolution ultérieure de la température (évolution due à des réactions chimiques exothermiques se produisant en arrière du front de détonation), fera l'objet d'une analyse plus détaillée.

5. Conclusion. - Encore que toutes les possibilités et caractéristiques de ce pyromètre n'aient pas encore fait l'objet d'une exploration systématique notamment en ce qui concerne sa précision et sa fidélité (cette dernière caractéristique pouvant être affectée par un éventuel vieillissement des fibres en particulier), il apparaît d'ores et déjà possible, en travaillant au-delà du seuil des $1700 \mathrm{~K}$, de détecter des variations de température mettant en jeu des temps très brefs de l'ordre d'une cinquantaine de nanosecondes, ce qui ouvre une large gamme de possibilités en matière de mesure de températures très rapidement variables. 


\section{Bibliographie}

[1] Ribaud, G., Traité de Pyrométrie optique, Rev. Opt. Théor. Instrum. 4 (1931.

[2] CRABOL, J., Solutions apportées à quelques problèmes posé par la détermination des températures de surface, Thèse de Doctorat ès Sciences Physiques, Paris, 1962.

[3] HornBeCK, G. A., Optical methods of temperature measurements, Appl. Opt. 5 (1966) 179-185.

[4] REYNolds, P. M., A review of multicolour pyrometry for temperatures below $1500^{\circ} \mathrm{C}$, Brit. J. Appl. Phys. 15 (1964) 579-589.

[5] Teychenne de Blazi, P., Malaval, C. et Lombard, J. M., Etude spectroscopique de la température de détonation d'un explosif contenant de l'aluminium, Centre d'Etude de Gramat, rapport final S 75-01, 30.9.1975.

[6] Demeter, L. et Childers, K., Optical pyrometer for submicrosecond temperature measurements, report number DNA-3177 T, October 1973.

[7] Gibson, F. C., Bowser, M. L., Summers, C. R., ScotT, F. H. et MAson, C. M., Use of an electro-optical method to determine detonation temperatures in high explosives. J. Appl. Phys. 29 (1958), 628-632.
[8] EISNER, L., Two color radiometer for use above $1000 \mathrm{~K}$, Adv. Test. Measur. Proc. 5th annual I. S. A. Symp., $\mathrm{n}^{\circ}$ 68-543, New-York, 1968.

[9] De vos, J. C., A new determination of the emissivity of tungsten ribbon, Physica, no 20 (1954) 690-714.

[10] Latyev, L. N., Chekhovskoi, V. Ya., Shestakov, E. N., Détermination expérimentale du pouvoir émissif du tungstène dans la région visible du spectre, dans la gamme de température $1200-2600 \mathrm{~K}$. Teplofizika Vysokikh Temperatur, 7 (1969) 666-673.

[11] Pyatr, E. C., Some consideration of the errors of brightness and two-colour types of spectral radiation pyrometer. Brit. J. Appl. Phys. 5 (1954) 264-268.

[12] Herne, H., The theoretical characteristics of bichromatic pyrometers, Brit. J. Appl. Phys. 4 (1953) 374-377.

[13] WiLson, R. G., Hemispherical spectral emittance of ablation chars, Carbon and Zirconia. Sumposium on thermal radiation of solids, San Francisco, Calif., March 4, $5,6,1964$. 